\title{
Necrotizing Fasciitis due to Candida Infection after Thyroid Surgery
}

\author{
Mustafa Aslier (D) \\ Case Report Department of Otorhinolaryngology Head and Neck Surgery, Sancaktepe Şehit Prof.Dr. İlhan Varank Training and Research \\ Hospital, İstanbul, Turkey
}

Abstract

ORCID ID of the author: M.A. 0000-0001-8257-0979.

Cite this article as: Aslier M. Necrotizing Fasciitis due to Candida Infection after Thyroid Surgery. Turk Arch Otorhinolaryngol 2020; 58(1):56-60.

Corresponding Author: Mustafa Aslıer; mustafa.aslier@gmail.com

Received Date: 15.11.2019 Accepted Date: 31.12 .2019 Available Online Date: 26.03 .2020

Content of this journal is licensed under a Creative Commons Attribution 4.0 International License. Available online at www.turkarchotolaryngol.net
Surgical site infections (SSIs) after thyroidectomy are very rare as it is a clean case surgery, even the patterns for the use of prophylactic antibiotics is disputable. Cutaneous and subcutaneous tissue infections are the most common type of SSIs that develop due to the contamination of the skin flora pathogens during surgical incision. We report the case of a patient who, unlike the reports in the literature, developed necrotizing fasciitis due to Candida infection after total thy- roidectomy. A 65-year-old male patient developed SSI progressing to necrotizing fasciitis four days after total thyroidectomy. Rapidly progressive necrosis was observed until antifungal treatment was initiated. Tissue defect eventually recovered with complete epithelization 40 days after the operation.

Keywords: Postoperative wound infection, thyroidectomy, Candida albicans, invasive fungal infections, necrotizing fasciitis

\section{Introduction}

Potential major complications of thyroid surgery include hypoparathyroidism, recurrent and superior laryngeal nerve paralysis, hemorrhage, seroma, skin scar and surgical site infections (SSIs) (1,2). As thyroidectomy is a clean case surgical procedure, SSIs are rare and routine antibiotic prophylaxis is not recommended (1, 2). Recent studies have reported $2 \%$ to $3.2 \%$ incidence of SSI in post-thyroidectomy patients. Conservative treatment with antibiotherapy is generally sufficient in the majority of the cases and almost no re-exploration is required $(3,4)$. In this case report, differently from the typical post-thyroidectomy SSIs in the literature, a case of SSI after thyroidectomy presented with necrotizing fasciitis (NF) due to candida infection is reported.

\section{Case Presentation}

A 65-year-old male patient was admitted to our outpatient clinic with a complaint of a swelling in his neck that had developed in the course of a year. Neck ultrasonography was performed and thyroid nodules were observed. The largest nodules were reported as $41 \mathrm{~mm}$ on the left and $24 \mathrm{~mm}$ on the right sides. The result of fine needle aspiration biopsy was reported as atypia of undetermined significance. Total thyroidectomy was recommended for the patient. No other pathological finding was identified in his medical history and in preoperative tests.

In the operation room $1 \mathrm{~g}$ IV cefazoline was administered for prophylaxis before the surgery. Thyroidectomy was performed successfully and bilateral parathyroid glands and laryngeal nerves were preserved. A drain was placed before surgical closure. Postoperatively, $1 \mathrm{~g}$ of amoxicillin clavulanic acid together with $500 \mathrm{mg}$ paracetamol was administered twice a day. On the first postoperative day $30 \mathrm{cc}$ of hemorrhagic fluid was measured in the drain and $20 \mathrm{cc}$ of serous-hemorrhagic drainage was observed on the second postoperative day; later on, the drainage catheter was removed. The patient was discharged on postoperative day 3 with $75 \mathrm{mcg}$ levothyroxine and ongoing oral antibiotherapy. 


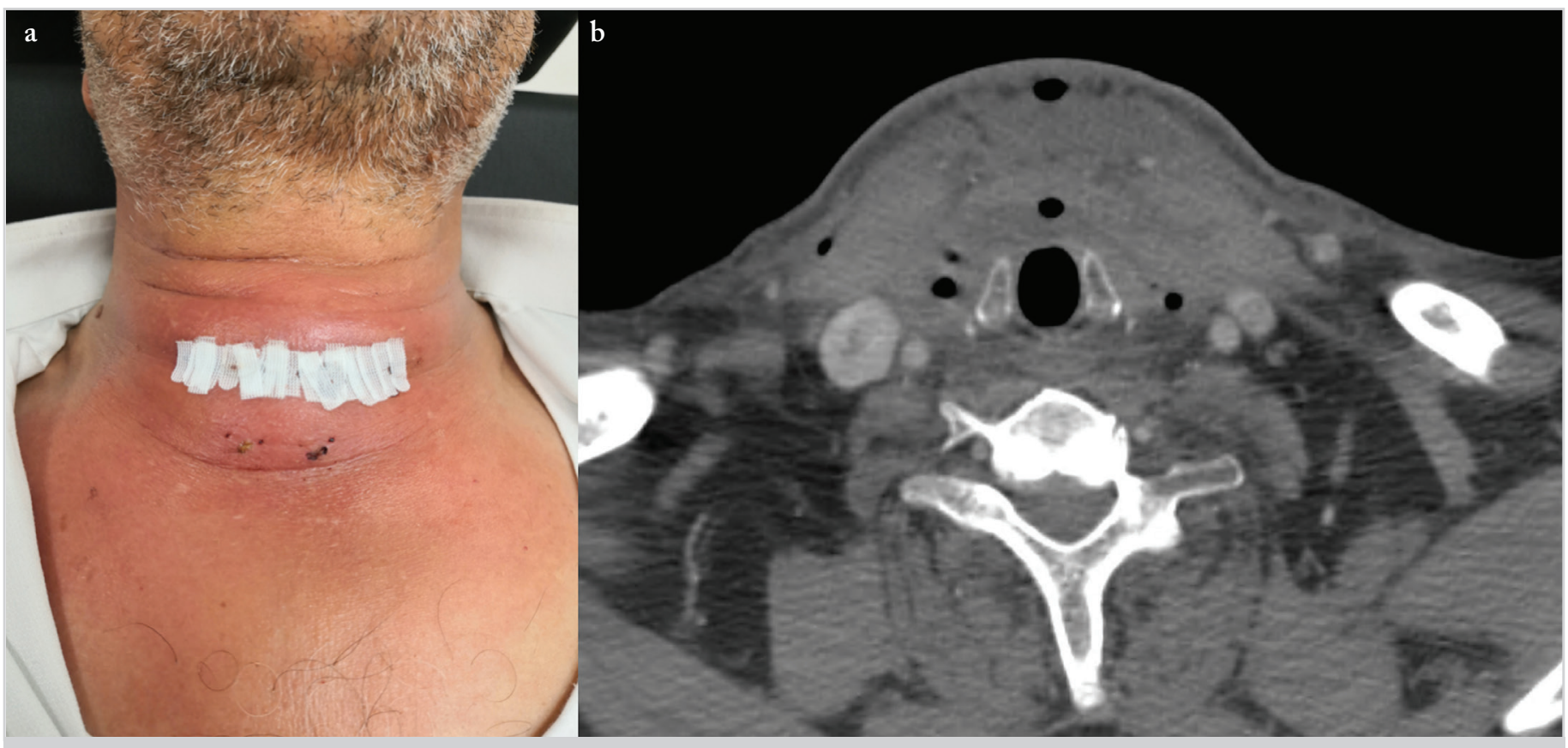

Figure 1. a, b. View of the neck of the patient at the time of admission to the emergency room four days after thyroidectomy. The skin was hyperemic, edematous and painful with fluctuating soft tissue swelling due to abscess (a). Axial plane CT image of the neck taken at the time of admission to the emergency room showed increased soft tissue density and localized air that were deemed compatible with edema and abscess in deep tissue (b)

The patient presented with a sudden swelling in his neck 24 hours after his discharge (postoperative day 4). In his physical examination, swelling and hyperaemia on the skin of surgical site was observed (Figure 1a). His body temperature was $39^{\circ} \mathrm{C}$. Complete blood count showed $81.6 \%$ neutrophil-dominancy and 21.4 103/uL leukocytosis; C-reactive protein (CRP) level was $20.77 \mathrm{mg} / \mathrm{dL}$. Contrast-enhanced neck computed tomography $(\mathrm{CT})$ scan revealed air densities and collections consistent with abscess content in the surgical site, the subcutaneous region, around the trachea and the adjacent soft tissue (Figure $1 \mathrm{~b})$. We made a puncture and $30 \mathrm{cc}$ purulent content was aspirated and sent to the microbiology laboratory for culture analysis. An antibiotherapy of $2 \mathrm{~g}$ ampicillin-sulbactam 4 times a day and $600 \mathrm{mg}$ clindamycin 3 times a day via intravenous route was begun after infectious diseases consultation.

The swelling in the neck recurred on the fifth day. The surgical site was explored under general anesthesia. The site was washed with physiological serum and drainage catheters were placed to maintain wound wash and dressings. Culture samples from the abscess content were repeatedly sent for microbiological analysis.

Black-colored skin necrosis started to develop around the drainage catheter and the incision line on the sixth day (Figure 2a). Drainage catheters were removed under sedation and necrotic tissues were debrided. Open wound care with hydrogen peroxide was started (Figure 2b). Ampicillin-sulbactam treatment was terminated upon the recommendation of the infectious diseases department and a daily dose of $4.5 \mathrm{~g}$ piperacillin-tazobactam divided into 3 times a day regimen was initiated.
Expansion was observed in the necrosis site on the seventh day (Figure 3a) and the clinical condition of the patient was evaluated in favor of NF. Elevated leukocytosis and CRP levels continued in blood tests. Piperacillin-tazobactam treatment was discontinued immediately after the recommendation of the infectious diseases department; clindamycin dose was increased to $900 \mathrm{mg} 3$ times a day, and in addition to that, $1 \mathrm{~g}$ vancomycin twice a day and $1 \mathrm{~g}$ imipenem 3 times a day were started. During daily examinations, the necrotic site was observed to expand within hours (Figure 3b). Laboratory reported that the microscopic examination of the tissue culture, collected the day before, revealed rare yeast hyphae formation and Candida albicans growth was identified in the fungal culture. Upon the results of culture work, anidulafungin with the loading dose of $200 \mathrm{mg}$ and maintenance dose of $100 \mathrm{mg}$ was added to the $\mathrm{pa}-$ tient's treatment with the recommendations of infectious diseases department. The reason to choose anidulafungin, which is more expensive in comparison to other antifungal drugs, was the association of elevated alanine aminotransferase (ALT) and aspartate aminotransferase (AST) levels of the patient.

Starting from the ninth day, patient's body temperature and leukocytosis began to decrease. Progression of the skin necrosis stopped (Figure 3c). Vancomycin treatment was discontinued on the $11^{\text {th }}$ day and clindamycin dose was reduced to $600 \mathrm{mg} 3$ times a day. Hospitalization of the patient was extended to 20 days and then the patient was discharged with medical treatment of $200 \mathrm{mg}$ fluconazole a day and $1 \mathrm{~g}$ oral amoxicillin clavulanic acid twice a day. Daily wound care was applied and the wound closure was let to secondary epithelization (Figure $3 \mathrm{~d}$ ). The patient finally became free of wound dressings 40 days after the operation and the tissue defect eventually recovered with complete epithelization (Figure 3e). At the end of the 
Aslier M.

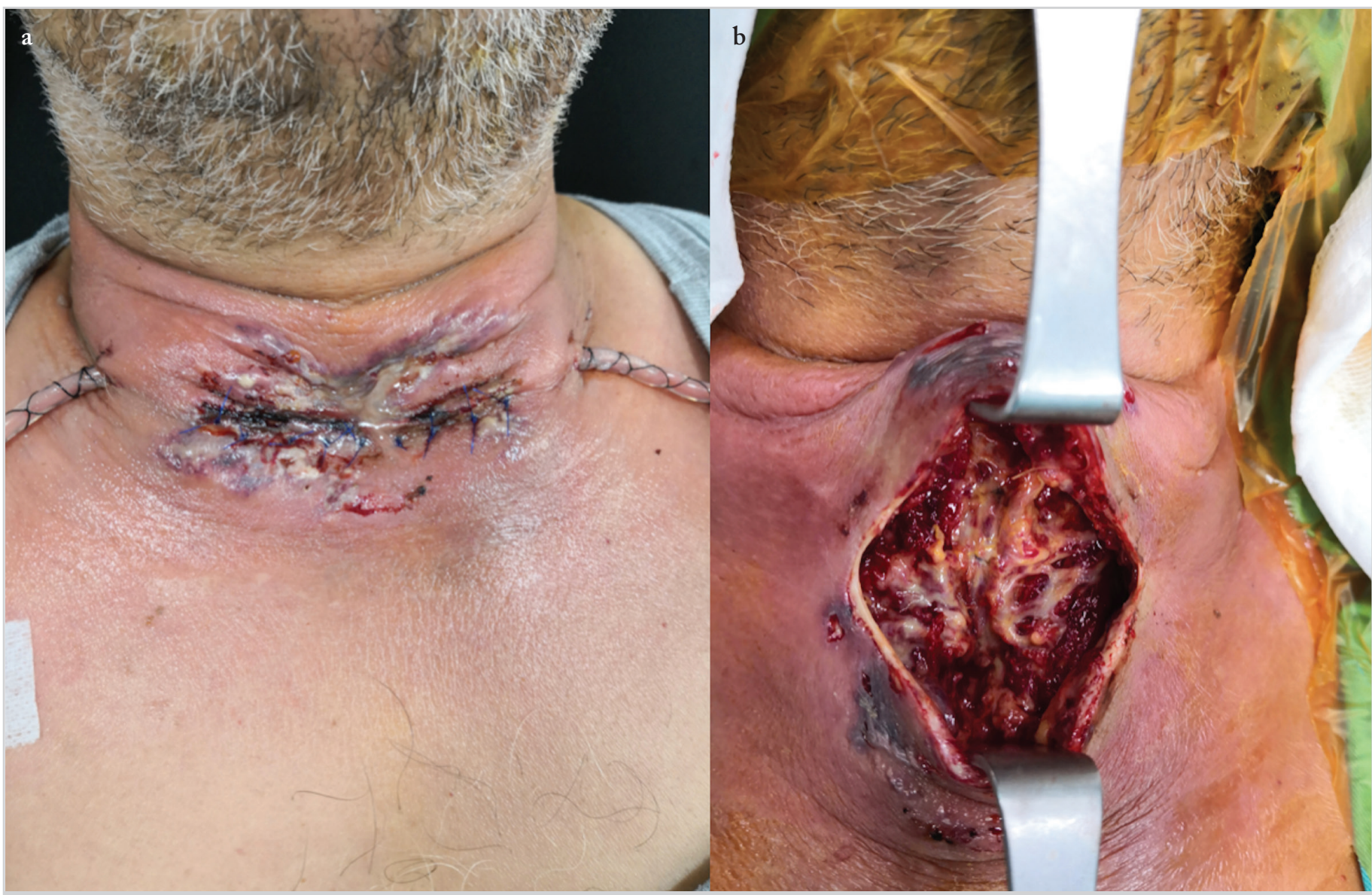

Figure 2. a, b. Black-colored skin necrosis around the drainage catheter and the incision line on the sixth day after neck exploration (a). Necrosis in the skin and the subcutaneous tissue on the sixth postoperative day during second neck exploration (b)
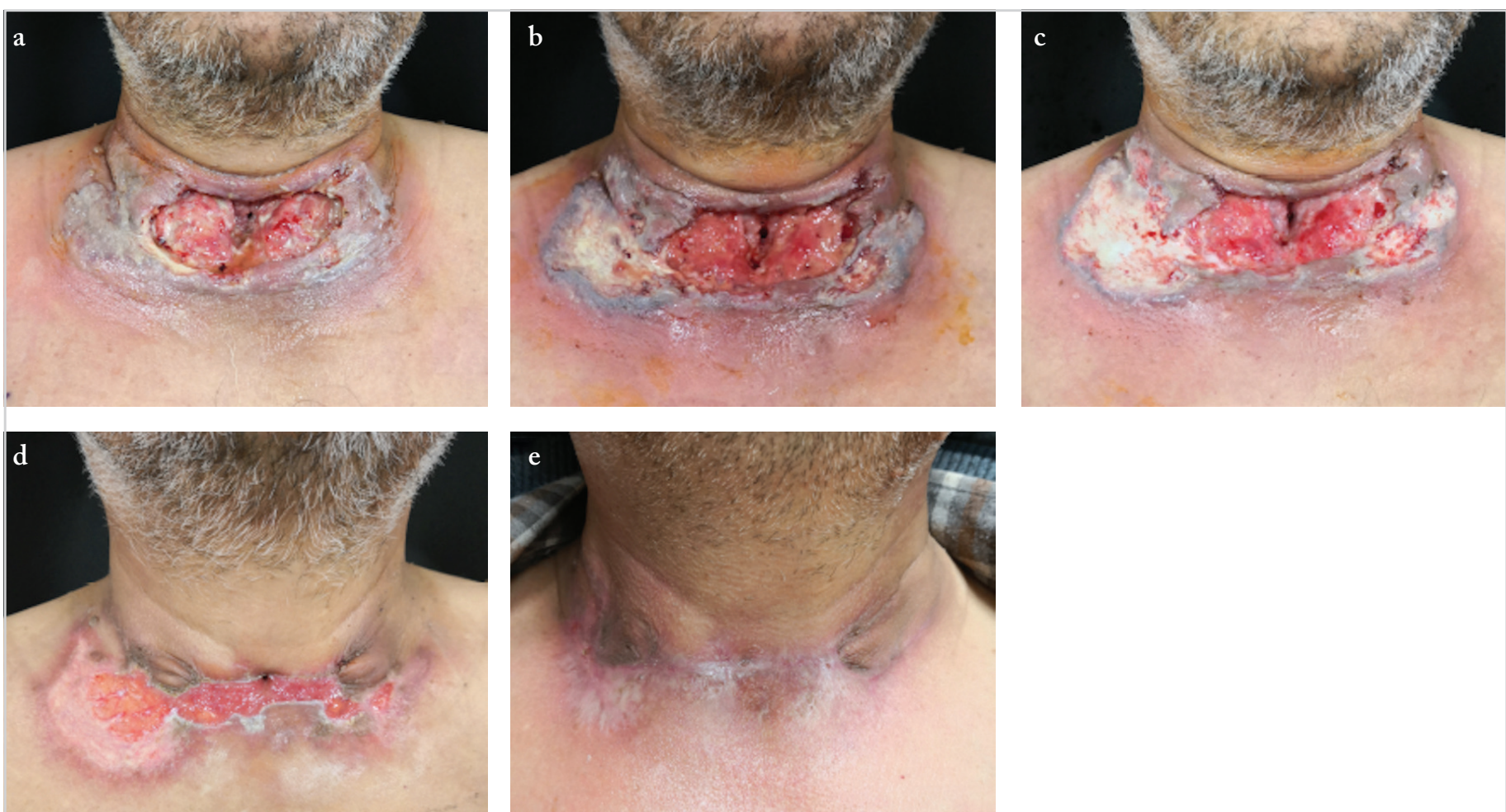

Figure 3. a-e. View of the wound defect with severe necrosis and fasciitis on the seventh postoperative day (a). View of the wound defect with severe necrosis and fasciitis surrounding with black-colored area on the eighth postoperative day (b). The surrounding black-colored area reduced with antifungal treatment on the ninth postoperative day (c). View of the epithelialization starting from the outside of the skin defect on the $20^{\text {th }}$ postoperative day (d). View of the neck after complete epithelization (e) 
whole treatment process, there was no permanent sequelae such as recurrent laryngeal nerve paralysis and hypoparathyroidism or hypocalcemia.

Informed consent was obtained from the patient for this report.

\section{Discussion}

Majority of the SSIs developing after thyroidectomy is limited to superficial tissue infection. Previous case series have shown that conservative treatment with appropriate antibiotics is sufficient in the presence of this type of SSIs (1-6). Our patient had diffused air and liquid densities that expanded within the neck fascia planes on postoperative day 4 . These findings, at the first admission, were found to be consistent with deep neck infection instead of superficial SSI. Therefore, follow-up and treatment algorithm of the patient was designed as deep neck infection treatment protocol.

Necrotizing fasciitis is a fatal infectious disease characterized by fast spreading tissue necrosis in subcutaneous tissue and fascia (7). Cervical NF is rare due to extensive vascular structures and tissue blood supply in the cervical region (7-9). Odontogenic infections (47\%) are the most common source of cervical NF and mortality rate is approximately 13\% (7). The presence of comorbid diseases like immunosuppression and diabetes mellitus are the leading risk factors. Our patient had no comorbid diseases or risk factors such as immunosuppression, diabetes mellitus or odontogenic infection. Although the typical pathogen is group A streptococci, it may develop as associated with $S$. aureus, E. coli, Pseudomonas spp., Bacteroides spp., and Klebsiella pneumonia or as a polymicrobial infection. Treatment consists of broad-spectrum antibiotherapy and surgical debridement as applied in the management of our patient. Since necrotic tissues act as a medium for microorganisms, extensive debridement should not be avoided. Hyperbaric oxygen treatment can be scheduled $(7,9)$.

Necrotizing fasciitis due to fungal infection is highly rare (8, 10). Although invasive fungus types are frequently isolated from cultures, candida species can also be a seldom factor. Penetration of microorganisms, which are already on normal skin flora, into the subcutaneous tissues during skin incision, direct contamination from surgical instruments due to inadequate sterilization, adhesions of fungal spores in the air to the surgical area and migration of microorganisms through the drain tubes are the potential transmission routes of candida infections $(8,10)$. We checked the sterilization process and no fault was detected. However, other factors could not be excluded.

It is a fast progressing condition characterized by air formation inside the soft tissue, similar to emphysematous gangrene as shown in the CT scan of our patient (Figure 2). Isolation of the microorganism in the culture, aggressive surgical debridement, broad-spectrum antibiotic therapy and treatment of comorbid diseases are required. If not identified, it has high mortality. Oc- casionally, wound closure challenges are encountered and the patient may be required to undergo reconstructive surgery after hemodynamical and clinical stability are achieved. However, the attentive care of the open wound with daily dressings and sterile bandage generally promotes complete secondary epithelization without the need of reconstruction with skin or soft tissue grafts and free flaps (7-10).

Our patient had a clinical course-similar to the few other cases published in the literature-that was characterized by fast spreading tissue necrosis which developed within hours. The clinical course of the patient improved to stable health conditions with the initiation of the appropriate antifungal and antibiotic treatment regimen immediately after the isolation of the responsible microorganism in the culture, and ongoing careful wound dressings.

\section{Conclusion}

Fungal infections must be considered among differential diagnosis of the patients who develop SSIs resembling an emphysematous gangrene clinic picture, causing tissue necrosis and do not respond to antibiotherapy after surgical procedures of the neck. In such cases, it is important that fungal elements are identified and fungal growth is detected in the samples during microbiological examination. Proper management of the patient by appropriate antifungal therapy together with meticulous wound care and dressings can prevent the patient from both a sepsis and a fatal clinical course.

Informed Consent: Written informed consent was obtained from the patient who participated in this case.

Peer-review: Externally peer-reviewed.

Conflict of Interest: The author has no conflicts of interest to declare.

Financial Disclosure: The author declared that this study has received no financial support.

\section{References}

1. Cannizzaro MA, Lo Bianco S, Picardo MC, Provenzano D, Buffone A. How to avoid and to manage post-operative complications in thyroid surgery. Updates Surg 2017; 69: 211-5. [Crossref]

2. Reeve T, Thompson NW. Complications of thyroid surgery: how to avoid them, how to manage them, and observations on their possible effect on the whole patient. World J Surg 2000; 24: 971-5. [Crossref]

3. Dionigi G, Rovera F, Boni L, Castano P, Dionigi R. Surgical site infections after thyroidectomy. Surg Infect (Larchmt) 2006; 7: 117-20. [Crossref]

4. Dionigi G, Rovera F, Boni L, Dionigi R. Surveillance of surgical site infections after thyroidectomy in a one-day surgery setting. Int J Surg 2008; 6: 13-5. [Crossref]

5. Owens CD, Stoessel K. Surgical site infections: epidemiology, microbiology and prevention. J Hosp Infect 2008; 70: 3-10. [Crossref]

6. Soylu E, Erdil A, Sapmaz E, Somuk BT, Akbulut N. Mediastinitis as complication of odontogenic infection: a case report. Niger J Clin Pract 2019; 22: 869-71. [Crossref]

7. Gunaratne DA, Tseros EA, Hasan Z, Kudpaje AS, Suruliraj A, Smith MC, et al. Cervical necrotizing fasciitis: systematic review 
Aslier M.

60 Necrotizing Fasciitis after Thyroid Surgery

and analysis of 1235 reported cases from the literature. Head Neck 2018; 40: 2094-102. [Crossref]

8. Abbasi Z, Inam H, Das S, Neel S, Fatimi SH. Fungal cervical abscess complicated by necrotizing fasciitis leading to descending necrotizing mediastinitis: a case report. Cureus 2019; 11: e5369. [Crossref]
9. Wang Y, Chen HC. Necrotizing fasciitis of the neck. Ear Nose Throat J. 2019 Sep 26. doi: 10.1177/0145561319874017. [Epub ahead of print] [Crossref]

10. Prasanna Kumar S, Ravikumar A, Somu L. Fungal necrotizing fasciitis of the head and neck in 3 patients with uncontrolled diabetes. Ear Nose Throat J 2014; 93: 18-21. 